\title{
Recurrent dislocation of binocular crystal lenses in a patient with cystathionine beta- synthase deficiency
}

\author{
Ning Hua ${ }^{1 *}$ D, Yuxian Ning ${ }^{1}$, Hui Zheng ${ }^{2}$, Ledong Zhao ${ }^{1}$, Xuehan Qian' ${ }^{1}$ Charles Wormington ${ }^{3}$ and Jingyun Wang ${ }^{4}$
}

\begin{abstract}
Background: Ectopia lentis is the common ocular manifestation of homocystinuria resulting from cystathionine beta-synthase (CBS) deficiency which has a high risk of thromboembolic complications.

Case presentation: The present study reports the case of a teenager with recurrent lens dislocation and glaucoma. He was diagnosed with CBS deficiency according to a high level of serum homocysteine and compound heterozygous mutations at two different positions on the CBS gene. Antiglaucoma eyedrops and a mydriatic successfully controlled the intraocular pressure, while oral pyridoxine and betaine uptake lowered the serum homocysteine level effectively.

Conclusions: Children with CBS deficiency may suffer from ectopia lentis, glaucoma and/or amblyopia. We firstly discovered a new mutation of CBS c. $697 \mathrm{~T}>\mathrm{G}$ which had not been reported before. The patient was pyridoxine responsive and well controlled by medicine.
\end{abstract}

Keywords: Case report, Cystathionine beta-synthase (CBS), Homocystinuria, Lens ectopia

\section{Background}

Homocystinuria due to cystathionine beta-synthase (CBS) deficiency, also known as classical homocystinuria (OMIM.org \#236200), is a rare recessive inherited amino acid metabolism disorder that involves the pathway for cystathionine synthesis. Patients with CBS deficiency manifest a variety of disorders involving multi-systems occurring from neonates to adults. The infantile presentation is the most clinically severe, and includes hypotonia, seizures, apneas and/or coma $[1,2]$. Adolescents or adults may present with intellectual disability/developmental delay, osteoporosis, psychiatric manifestations, and 'marfanoid' habitus $[1,3,4]$. Ocular manifestation mainly

\footnotetext{
* Correspondence: tian2815@sina.com

'Tianjin Key Laboratory of Retinal Functions and Diseases, Tianjin Branch of National Clinical Research Center for Ocular Disease, Eye Institute and School of Optometry, Tianjin Medical University Eye Hospital, No. 251 Fukang Road, Nankai District, Tianjin, China

Full list of author information is available at the end of the article
}

includes crystalline lens dislocation, which might be misdiagnosed as Marfan's syndrome. However, CBS deficiency is highly correlated with thrombosis, which is the main cause of mortality [3]. The proper diagnosis is vital for clinicians to provide reasonable management.

We herein report a case of a 14-year-old boy with CBS deficiency presenting with recurrent dislocation of the crystalline lens from the anterior chamber to the vitreous cavity. In addition, we found compound heterozygous mutations at two different positions on the CBS gene. One of the genetic variants, c.697 T > G (p. W233N), has not been reported before.

\section{Case presentation}

A 14-year-old boy was referred to the Department of Pediatric Ophthalmology and Strabismus at Tianjin Medical University Eye Hospital due to conjunctiva injection at the left eye with blurring for nearly 1 month. He had severe myopia with poorly corrected vision at a

(C) The Author(s). 2021 Open Access This article is licensed under a Creative Commons Attribution 4.0 International License, which permits use, sharing, adaptation, distribution and reproduction in any medium or format, as long as you give appropriate credit to the original author(s) and the source, provide a link to the Creative Commons licence, and indicate if changes were made. The images or other third party material in this article are included in the article's Creative Commons licence, unless indicated otherwise in a credit line to the material. If material is not included in the article's Creative Commons licence and your intended use is not permitted by statutory regulation or exceeds the permitted use, you will need to obtain permission directly from the copyright holder. To view a copy of this licence, visit http://creativecommons.org/licenses/by/4.0/ The Creative Commons Public Domain Dedication waiver (http://creativecommons.org/publicdomain/zero/1.0/) applies to the data made available in this article, unless otherwise stated in a credit line to the data. 
very young age. Since being a toddler, he was always the tallest in classes. However, the parents complained about their son's bad temper and academic retardation. Seizures or thrombosis were denied. The boy had a 'marfanoid' habitus with scoliosis and arachnodactyly.

The uncorrected visual acuity was 6/120 in the right eye and finger counting in the left eye. The crystalline lenses were located in the anterior chamber binocularly, while the intraocular pressure was $45 \mathrm{mmHg}$ in the left eye with conjunctiva injection (Fig. 1a), and $16 \mathrm{mmHg}$ in the right eye (Fig. 1b). Simultaneously, bilateral iris atrophy around the pupil was detected.

The initial management included anti-glaucoma eye drops, carteolol hydrochloride $2 \%$ and brinzolamide $1 \%$ for daily use, and oral methazolamide $25 \mathrm{mg}$ Q8H. The intraocular pressure became normal with the disappearance of corneal edema in the left eye after 2 days of treatment. (Fig. 1c).

In addition, the crystalline lens in the right eye was found to be in the inferior vitreous cavity (Fig. 1d) with a strand of elongated zonular fibers at 12 o'clock to the ciliary body. The refractive error was $+12.00 /-2.00 \times 80(\mathrm{SE}+11.00 \mathrm{D})$ in the right eye with the lens in the inferior vitreous, and $18.00 /-4.00 \times 30$ (SE - 20.00D) in the left eye with the lens in the anterior chamber. The best-corrected visual acuity was $6 / 12$ in the right eye and 6/30 in the left eye.
Since then, careful slit lamp examination showed the recurrent changes of the optic lens from the vitreous cavity to the anterior chamber during 5 days of observation. There were several lens position changes in a single day, accompanied with a posture change from the supine position.

The plasma homocysteine concentration was $309.6 \mu \mathrm{mol} / \mathrm{L}$, over 20 times the normal laboratory level (under $15 \mu \mathrm{mol} / \mathrm{L}$ ). Whole exome sequencing and Sanger sequencing for the genetic variants (GBI Shenzhen, China) was performed from the blood samples of the patient and his parents. The result showed gene mutations in the CBS gene at two different positions, NM 000071.2(CBS): c. 502G > A, and NM_000071.2(CBS): c. $697 \mathrm{~T}>\mathrm{G}$ (Table 1). The mutation c.502G > A was inherited from his father, who was heterozygous, while the mutation c. $697 \mathrm{~T}>\mathrm{G}$ was inherited from the heterozygous mother (Fig. 2). Although the parents had different mutations in the two positions of the CBS gene, both were normal not only in general systems but also in eyes with normal vision.

Therefore, the boy was diagnosed with the CBS deficiency and treated with a supplement of pyridoxine $50 \mathrm{mg}$ daily, betaine $3.0 \mathrm{~g}$ bid and a low-Met diet in another children's hospital. The ocular surgery was delayed because of the high risk of thrombosis.
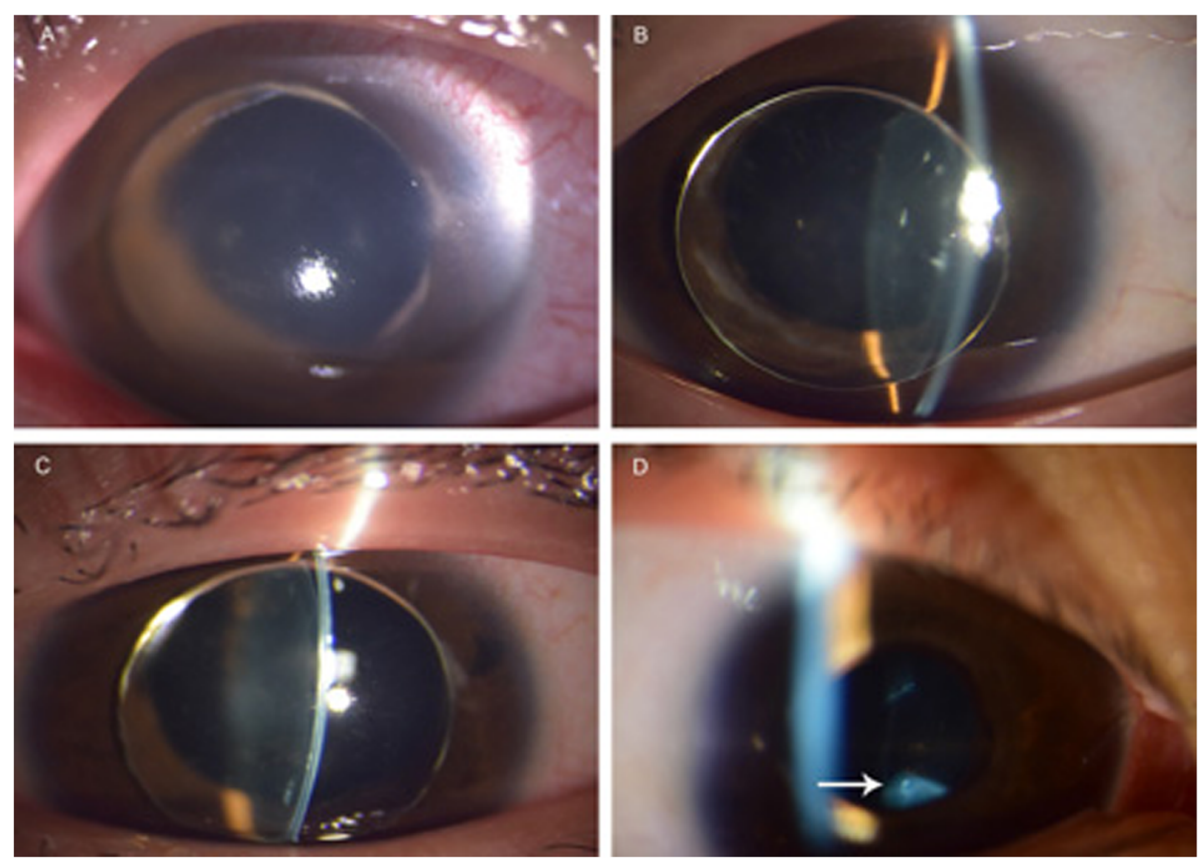

Fig. 1 The changes of the lens location during medical control of intraocular pressure. a. The crystalline lens was totally dislocated into the anterior chamber in the left eye with corneal edema due to pupillary-block glaucoma; the intraocular pressure was 45 mmHg. $\mathbf{b}$. The same lens dislocated in the right eye with normal intraocular pressure of $16 \mathrm{mmHg}$; the iris atrophy was clearly observed around the pupil. c. With control of the pressure, the corneal edema disappeared in the left eye and the lens was still in the anterior chamber. Marginal atrophy around the pupil was apparent. $\mathbf{d}$. After the patient stood up from lying on his back, the spontaneous backwards movement of lens into the vitreous cavity in the left eye was detected. The white arrow indicated the dislocated lens in the inferior vitreous cavity 
Table 1 Gene detections of the patient and his parents

\begin{tabular}{|c|c|c|c|c|c|c|c|}
\hline Gene detection & Gene & Exon & Genome coordinates & Nucleotide change & AA change & Hom/Hct/Hem & References \\
\hline \multirow[t]{2}{*}{ The patient } & CBS & Exon8 & chr21:44485352 & c.697T > G & p.Tyr233Asp & $\mathrm{Hct}$ & - \\
\hline & CBS & Exon6 & chr21:44485755 & C. $502 \mathrm{G}>\mathrm{A}$ & p.Val168Met & $\mathrm{Hct}$ & 16 \\
\hline \multirow[t]{2}{*}{ The parents } & \multicolumn{5}{|c|}{ Mother of the patient } & \multicolumn{2}{|l|}{ c.697 T > G } \\
\hline & \multicolumn{5}{|c|}{ Father of the patient } & \multicolumn{2}{|l|}{$c .502 \mathrm{G}>\mathrm{A}$} \\
\hline
\end{tabular}

18B4028977-1235-FO2_CBSCds6R_F11

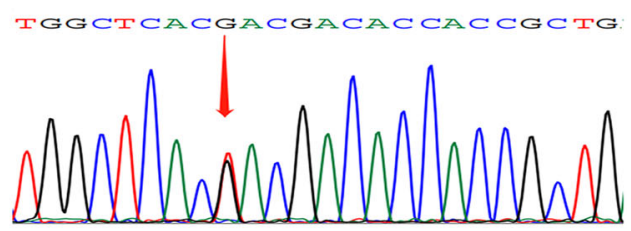

18B4028978-1235-GO2_CBSCds6R G11

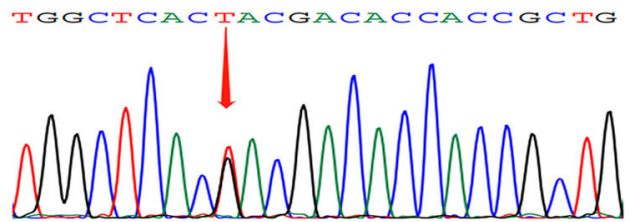

18B4028979-1235-HO2_CBScds6R_H11

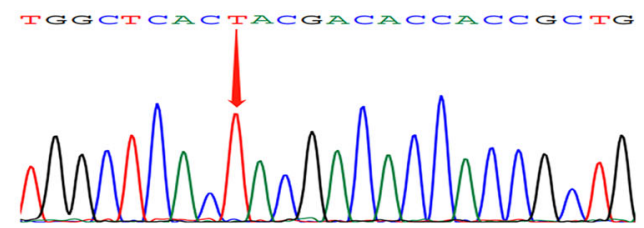

CBS;NM_000071.2;c.697T>G;p.Tyr233Asp

18B4028977-1215-AO4_CBScds4R_A12

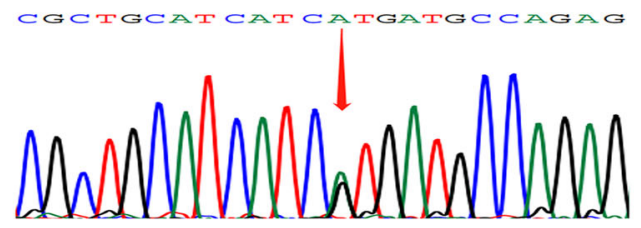

18B4028978-1215-BO4_CBScds4R_B12

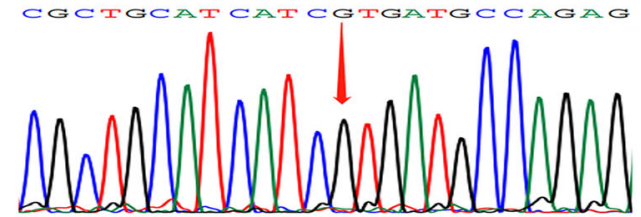

18B4028979-1215-CO4_CBScds4R

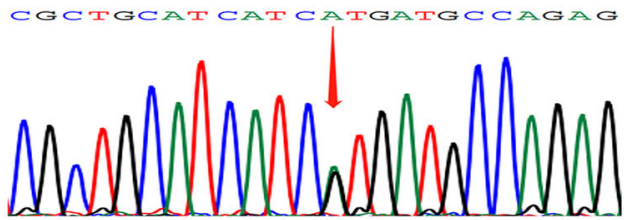

CBS;NM_000071.2;c.502G>A;p.Val168Met

Fig. 2 Sanger sequencing of the patient and the parents. Sample 18B4028977 was from the patient, $18 B 4028978$ was from the mother and 18 B4028979 was from the father 
At the three-month follow-up, the plasma homocysteine (Hcy) concentration was lowered to $75.0 \mu \mathrm{mol} / \mathrm{L}$. The lens located in the inferior vitreous cavity while the pupils were mildly dilated in both eyes with normal intraocular pressure $(15 \mathrm{mmHg}$ in the right eye, $14 \mathrm{mmHg}$ in the left eye). The best-corrected vision was $6 / 9.5$ in the right eye $(+12.00 /-2.00 \times 80, \mathrm{SE}+11.00 \mathrm{D}), 6 / 24$ in the left eye $(+11.00 /-1.50 \times 100$, SE +10.25D).

Pyridoxine, betaine as well as diet restriction was continued to reach the optimal target for the plasma Hcy at $<50 \mu \mathrm{mol} / \mathrm{L}$ [1]. To prevent pupillary-block glaucoma, mydriasis with tropicamide eye drops was suggested. The further plan included pars plana lensectomy combined with posterior chamber scleral fixation of an intraocular lens after the plasma Hcy was well controlled at the normal level. Low molecular weight heparin anticoagulation would be required preoperatively.

\section{Discussion}

Homocysteine (Hcy) is formed in the catabolic pathway for methionine (Met), an essential amino acid taken up from animal protein digestion [5]. CBS deficiency impairs the conversion of Hcy to cystathionine and leads to Hcy and Met accumulation. The pathophysiology of CBS deficiency is not fully understood. It is suggested that elevated Hcy concentrations modify sulfhydryl groups on proteins and interfere with the cross-linking of sulfhydryl groups in proteins such as elastin, which is considered to lead to the ectopia lentis and skeletal abnormalities [4]. Elevated Hcy concentrations also alter intracellular signaling and cause endoplasmic reticulum stress, which can result in thromboembolism and vascular disease $[6,7]$.

A low-Met diet and supplement of pyridoxine (vitamin $B_{6}$ ) were prescribed to lower the Hcy concentration as close to the normal level as possible. Early-diagnosed patients have opportunities to maintain normal growth and to avoid severe complications $[8,9]$. For late-diagnosed patients, reasonable control of serum homocysteine level can reduce the risk of further complications, such as seizures, abnormal behaviors, and thromboembolic disease [10-12].

However, it is not every patient that is sensitive to the supplement of pyridoxine. It depends on the location of mutations on the CBS gene. To date, over 900 CBS alleles in patients of varied ethnic origins were characterized and 164 different pathogenic genetic variants were observed [13]. Only a few mutations present in the homozygous state were shown to have genotype/phenotype correlations, concordance between pyridoxine responsiveness and clinical phenotype.

Some specific mutations cause a severe pyridoxine nonresponsive form of the disease when inherited in the homozygous state, such as the c.919G > A (p.G 307S) tested in Ireland, the c.572C > T (p.T191M) in Spain, Portugal and South America $[14,15]$. On the contrary, the c.833 T > C (p.I278T) variant, which was derived from Europeans, manifested a mild pyridoxine-responsive type of CBS deficiency when homozygous [13]. Compound heterozygotes carrying the c.833 T > C (p. I278T) variant on one allele are partially pyridoxine-responsive in some patients, whereas totally not responsive in others $[14,15]$. Therefore, such correlations are difficult to infer in individuals who are compound heterozygotes.

In this case of CBS deficiency, the CBS gene mutated at two different positions, NM_000071.2(CBS): c. 502G > A, and NM_000071.2(CBS): c.697 T > G. The missense mutation c.502G $>$ A was reported as a pathogenic mutation [16]. But paternal manifestation was clinically negative. This was probably due to heterozygosity at the CBS gene. For c. $697 \mathrm{~T}>\mathrm{G}$ (p. W233N) in the proband, which was inherited from his asymptomatic mother, there was no previous literature report. It was confirmed by Sanger sequencing of samples from the patient and his mother. The nearby missense variants (p. A231P, p. H232D, p. D234N, p. E239K) have been reported in the Human Gene Mutation Database in association with homocystinuria, supporting the functional importance of this region of the protein [17]. The result of Condel, SIFT and PolyPhen also supported that the missense single nucleotide variant was deleterious for protein activity [18-20].

We found that our patient was a compound heterozygote at c. 502G > A and c. $697 \mathrm{~T}>$ G. Fortunately, after 3 months of diet control of Met and supplement of pyridoxine, his plasma Hcy concentration was lowered to $75.0 \mu \mathrm{mol} / \mathrm{L}$, which met the criterion for metabolic control $[1,3]$. The result indicated that this compound heterozygote was pyridoxine-responsive.

In this case, the ectopia lentis significantly manifested as the lens moved back and forth between the inferior vitreous cavity and the anterior chamber. The recurrent movements of the lens apparently induced mechanical injury to the iris, resulting in iris atrophy.

The blockage of aqueous humor due to dislocated lens in the anterior chamber led to the acute elevation of intraocular pressure when the pupil was not large enough. Furthermore, the frequent changes of lens position caused significant uncorrected refractive fluctuations, which happened from a very young age resulting in amblyopia. When the lens dislocated into the inferior vitreous cavity, the refraction was high hyperopia due to the absence of lens refractive power. However, it changed into severe myopia when the lens moved forwards into the anterior chamber, thus moving the focal point in front of the retina significantly. Frame glasses could not precisely correct such dramatically changing refractive errors. Thus, amblyopia could be developed due to the refractive fluctuations [21]. 
The most effective treatment might be surgically removing the lens with intraocular lens implantation [21-25]; therefore, the postoperative refraction could facilitate amblyopia treatment. Meanwhile, the replacement of the recurrent dislocated lens with an IOL might effectively eliminate the recurrence of pupillary block glaucoma. However, the parents could not bear the risk of thrombosis as a complication of surgery. Therefore, we arranged a reasonable medical treatment, including mydriatic and anti-glaucoma eye drops; and the intraocular pressure was carefully monitored at each follow-up. If the boy accepts lensectomy with IOL implantation, the visual acuity may improve gradually to a satisfactory level; as a teenager, he still has a potential to acquire better vision with reasonable amblyopia treatments.

Although patients with Marfan's syndrome usually demonstrate a tall and thin appearance with arachnodactyly, the direction of partial lens dislocation is commonly supertemporal, which may be detected as the upper dislocation of the lens with the margin being observed at the pupil through the slit lamp examination [26]. However, the typical ocular manifestation of the CBS deficiency is a total lens dislocation in the anterior chamber, or sinking into the lower part of the vitreous cavity [1]. If a child is referred to an ophthalmologist with lens dislocation, the serum homocysteine concentration should be tested when the traumatic injury is excluded. Since CBS deficiency is an autosomal recessive condition, family members at risk for the disease should be tested by measuring Hcy or, in exceptional cases, by molecular genetic or enzymatic analysis.

In this case, the metabolic control did not affect the eye structure nor the refraction. The dislocated lens did not change due to the rupture of most of the ciliary zonules. However, for early diagnosed patients without complications, the metabolic control treatment can realistically aim to prevent all the complications of CBS deficiency including ectopia lentis, whilst maintaining normal growth and nutrition [1]. An enzyme replacement therapy in the mice model successfully demonstrated that the early control (initiated 3 weeks of age and continued for a further 9 months) of plasma Hcy prevented the rupture of the ciliary zonules, thus rescued the ocular phenotype [27]. For late-diagnosed patients, the aim is to prevent further complications, especially thromboembolic disease. Raised Hcy concentrations cause endothelial dysfunction as well as impaired thrombolysis, which may be responsible for thromboembolism and vascular disease. Sound control of plasma Hcy is effective to lower the risk of thrombosis and other complications.

Dehydration and infection increase the risk of venous thrombosis, particularly in children $[28,29]$. It is important to ensure patients with CBS deficiency are wellhydrated at all times, especially when sick and during anesthesia and surgery.
Monitoring of plasma Hcy, AA, folate and vitamin B12 is recommended in all patients [1]. Patients on dietary treatment require regular nutritional assessment and additional tests to avoid poor growth or malnutrition. Restricting intake of Met reduces Hcy production. However, children with severe CBS deficiency still require a low-Met intake to maintain normal growth [30].

Bone density scans (DEXA) should be tested every 35 years from adolescence [31]. Vitamin D and calcium supplements should be prescribed if osteoporosis exists.

For long-term uptake of pyridoxine and betaine, adverse effects such as peripheral neuropathy should be monitored in the regular follow-up [32].

As cystathionine beta-synthase resides in the liver, liver transplant has been performed and manifested as an effective treatment. Some cases of liver transplant were reported with successful metabolic control without any dietary restrictions [33-35]. Meanwhile, research indicates that livertargeted gene therapy has effectively lowered the concentration of Hcy in a CBS-deficiency mouse model, bringing hope for the possible treatment of the disease [36, 37].

\section{Conclusion}

Children with CBS deficiency may suffer from ectopia lentis, glaucoma and/or amblyopia. The gene detection in this case showed a mutation at c.697 T $>$ G, which was never reported before. The patient was pyridoxine responsive and well controlled by medicine.

\section{Acknowledgments \\ The authors thank the parents and the patient for their generous agreement for the publication of the study. The authors also thank Dr. Song Lin (Tianjin Medical University Eye Hospital) for taking excellent anterior segment photos and Dr. Huiyu Wang (Tianjin Medical University Eye Hospital) for measurement of refractive errors respectively.}

\section{Authors' contributions}

$\mathrm{NH}$ drafted the manuscript and reviewed the literature. $\mathrm{YN}$ and $\mathrm{HZ}$ collected the data. LDZ and XHQ interpreted the data and reviewed the literature. CW and JW were involved the design and helped to draft the manuscript. All authors read and approved the final manuscript.

\section{Funding}

This work received funding support from Science and technology projects in the field of social development in Tianjin Binhai New Area (BHXQKJXM-SF2018-04) and Tianjin key clinical discipline (specialty) construction project (TJLCZDXKM001)

\section{Availability of data and materials}

The genetic datasets generated and/or analysed during the current study are available in the CNGB Sequence Archive of China National GeneBank

DataBase repository, accession number CNP0001806.

\section{Declarations}

Ethics approval and consent to participate

The study was approved by the Institutional Ethics Board of Tianjin Medical University Eye Hospital and followed the Declaration of Helsinki. The patient and his parents all signed the written consent forms to participate in the study. 


\section{Consent for publication}

Written informed consent was obtained from the patient and his parents for publication of this case report and images. A copy of the written consent is available for review by the Editor of this journal.

\section{Competing interests}

The authors declare that they have no competing interests.

\section{Author details}

'Tianjin Key Laboratory of Retinal Functions and Diseases, Tianjin Branch of National Clinical Research Center for Ocular Disease, Eye Institute and School of Optometry, Tianjin Medical University Eye Hospital, No. 251 Fukang Road, Nankai District, Tianjin, China. ${ }^{2}$ Tianjin Eye Hospital, Tianjin, China. ${ }^{3}$ Salus Univerisity Pennsylvania College of Optometry, Elkins Park, PA, USA. ${ }^{4}$ SUNY College of Optometry, New York, NY, USA.

\section{Received: 18 October 2020 Accepted: 5 May 2021}

\section{Published online: 13 May 2021}

\section{References}

1. Morris AA, Kožich V, Santra S, Andria G, Ben-Omran TI, Chakrapani AB, et al. Guidelines for the diagnosis and management of cystathionine betasynthase deficiency. J Inherit Metab Dis. 2017;40(1):49-74.

2. Cruysberg JR, Boers GH, Triibels JM, Deutman AF. Delay in diagnosis of homocystinuria: retrospective study of consecutive patients. BMJ. 1996; 313(7064):1037-40.

3. McCully KS. Homocysteine and the pathogenesis of atherosclerosis. Expert Rev Clin Pharmacol. 2015;8(2):211-9.

4. Abbott MH, Folstein SE, Abbey H, Pyeritz RE. Psychiatric manifestations of homocystinuria due to cystathionine beta-synthase deficiency: prevalence, natural history, and relationship to neurologic impairment and vitamin B6responsiveness. Am J Med Genet. 1987;26(4):959-69.

5. Perry DJ. Hyperhomocysteinaemia. Baillieres Best Pract Res Clin Haematol. 1999;12(3):451-77.

6. Lai WK, Kan MY. Homocysteine-induced endothelial dysfunction. Ann Nutr Metab. 2015;67(1):1-12.

7. Schienle HW, Seitz R, Nawroth P, Rohner I, Lerch L, Krumpholz B, et al. Thrombomodulin and ristocetincofactor in homocystinuria: a study in two siblings. Thromb Res. 1995;77(1):79-86.

8. Yap S. Classical homocystinuria: vascular risk and its prevention. J Inherit Metab Dis. 2003;26(2-3):259-65.

9. Wilcken DE, Wilcken B. The natural history of vascular disease in homocystinuria and the effects of treatment. J Inherit Metab Dis. 1997;20(2): 295-300.

10. Yap S, Naughten ER, Wilcken B, Wilcken DE, Boers GH. Vascular complications of severe hyperhomocysteinemia in patients with homocystinuria due to cystathionine beta-synthase deficiency: effects of homocysteine-lowering therapy. Semin Thromb Hemost. 2000;26(3):335-40.

11. Kabra M. Dietary management of inborn errors of metabolism. Indian J Pediatr. 2002;69(5):421-6

12. Garland J, Prasad A, Vardy C, Prasad C. Homocystinuria: challenges in diagnosis and management. Paediatr Child Health. 1999:4(8):557-62.

13. Skovby F, Gaustadnes M, Mudd SH. A revisit to the natural history of homocystinuria due to cystathionine beta-synthase deficiency. Mol Genet Metab. 2010:99(1):1-3.

14. Cozar M, Urreizti R, Vilarinho L, Grosso C, Dodelson de Kremer R, Asteggiano CG, et al. Identification and functional analyses of CBS alleles in Spanish and Argentinian homocystinuric patients. Hum Mutat. 2011;32(7):835-42.

15. Alcaide $P$, Krijt J, Ruiz-Sala P, Ješina P. Ugarte M, Kožich V, et al. Enzymatic diagnosis of homocystinuria by determination of cystathionine- $B$-synthase activity in plasma using LC-MS/MS. Clin Chim Acta. 2015:438:261-5.

16. Kruger WD, Cox DR. A yeast assay for functional detection of mutations in the human cystathionine beta-synthase gene. Hum Mol Genet. 1995;4(7): 1155-61.

17. Stenson PD, Mort M, Ball EV, Shaw K, Phillips A, Cooper DN. The human gene mutation Database: building a comprehensive mutation repository for clinical and molecular genetics, diagnostic testing and personalized genomic medicine. Hum Genet. 2014;133(1):1-9.

18. Gonzalez-Perez A, Lopez-Bigas N. Improving the assessment of the outcome of nonsynonymous SNVs with a consensus deleteriousness score. Condel Am J Hum Genet. 2011;88(4):440-9.
19. Ng PC, Henikoff S. Predicting deleterious amino acid substitutions. Genome Res. 2001:11(5):863-74.

20. Adzhubei IA, Schmidt S, Peshkin L, Ramensky VE, Gerasimova A, Bork P, et al. A method and server for predicting damaging missense mutations. Nat Methods. 2010;7(4):248-9.

21. Neely DE, Plager DA. Management of ectopia lentis in children. Ophthalmol Clin N Am. 2001;14(3):493-9.

22. Babu N, Muraly P, Ramasamy K. Twenty-three-gauge two-port pars plana lensectomy for the management of ectopia lentis in children. Retina. 2010; 30(6):971-4

23. Behki R, Noel LP. Clarke WN. Limbal lensectomy in the management of ectopia lentis in children. Arch Ophthalmol. 1990;108(6):809-11.

24. Kemmanu V, Rathod P, Rao HL, Muthu S, Jayadev C. Management of cataracts and ectopia lentis in children: practice patterns of pediatric ophthalmologists in India. Indian J Ophthalmol. 2017;65(9):818-25.

25. Shortt AJ, Lanigan B, O'Keefe M. Pars plana lensectomy for the management of ectopia lentis in children. J Pediatr Ophthalmol Strabismus. 2004;41(5):289-94

26. Konradsen TR, Zetterstrom C. A descriptive study of ocular characteristics in Marfan syndrome. Acta Ophthalmol. 2013;91(8):751-5.

27. Majtan T, Jones W Jr, Krijt J, Park I, Kruger WD, Kožich V, et al. Enzyme replacement therapy ameliorates multiple symptoms of murine Homocystinuria. Mol Ther. 2018;26(3):834-44.

28. Baddouh N, Elbakri S, Draiss G, Mouaffak Y, Rada N, Younous S, et al. Cerebral venous thrombosis in children: about a series of 12 cases. Pan Afr Med J. 2019:32:22

29. Eslamiyeh H, Ashrafzadeh F, Akhondian J, Beiraghi TM. Homocystinuria: a rare disorder presenting as cerebral Sinovenous thrombosis. Iran J Child Neurol. 2015;9(2):53-7.

30. Purcell O, Coughlan A, Grant T, McNulty J, Clark A, Deverell D, et al. Growth patterns in the Irish pyridoxine nonresponsive homocystinuria population and the influence of metabolic control and protein intake. J Nutr Metab. 2017:2017:8570469

31. Weber DR, Coughlin C, Brodsky JL, Lindstrom K, Ficicioglu C, Kaplan P, et al. Low bone mineral density is a common finding in patients with homocystinuria. Mol Genet Metab. 2016;117(3):351-4.

32. Echaniz-Laguna A, Mourot-Cottet R, Noel E, Chanson JB. Regressive pyridoxine-induced sensory neuronopathy in a patient with homocystinuria. BMJ Case Rep. 2018:2018:bcr2018225059.

33. Snyderman SE. Liver failure and neurologic disease in a patient with homocystinuria. Mol Genet Metab. 2006;87(3):210-2.

34. Lin NC, Niu DM, Loong CC, Hsia CY, Tsai HL, Yeh YC, et al. Liver transplantation for a patient with homocystinuria. Pediatr Transplant. 2012 16(7):E311-4.

35. Kerkvliet SP, Rheault MN, Berry SA. Liver transplant as a curative treatment in a pediatric patient with classic homocystinuria: a case report. Am J Med Genet A. 2021;185(4):1247-50.

36. Lee HO, Gallego-Villar L, Grisch-Chan HM, Häberle J, Thöny B, Kruger WD. Treatment of cystathionine beta-synthase deficiency in mice using a minicircle-based naked DNA vector. Hum Gene Ther. 2019;30(9):1093-100.

37. Baloula V, Fructuoso M, Kassis N, Gueddouri D, Paul JL, Janel N. Homocysteine-lowering gene therapy rescues signaling pathways in brain of mice with intermediate hyperhomocysteinemia. Redox Biol. 2018;19:200-9.

\section{Publisher's Note}

Springer Nature remains neutral with regard to jurisdictional claims in published maps and institutional affiliations. 